\title{
MYRICETIN ISOLATED FROM TURBINARIA ORNATA AMELIORATES ROTENONE INDUCED PARKINSONISM IN DROSOPHILA MELANOGASTER
}

\author{
VIJAYRAJA DHANRAJ ${ }^{1}$, TAMILARASAN MANIVASAGAM ${ }^{2}$, JEYAPRAKASH KARUPPAIAH ${ }^{3}$
}

${ }^{1}$ Research and Development Centre, Bharathiar University, Coimbatore 641046, Tamilnadu, India, ${ }^{2}$ Department of Biochemistry and Biotechnology, Annamalai University, Chidambaram 608002, Tamil Nadu, India, ${ }^{3}$ PG and Research Department of Biochemistry, Rajah Serfoji Government College, Thanjavur 613005, India

Email: jeypee5@gmail.com

Received: 13 May 2017 Revised and Accepted: 21 Sep 2017

\section{ABSTRACT}

Objective: Parkinson's disease (PD) is a neurodegenerative disorder which affects the elderly population. Free radicals overproduction, oxidative stress, apoptosis, inflammation and abnormalities in mitochondria are critical mediators of the neuronal degeneration. In the present study neuroprotective activity of myricetin, a flavonoid isolated from brown seaweed Turbinaria ornata have been investigated in rotenone induced experimental PD models of Drosophila melanogaster.

Methods: Male fruit flies (Drosophila melanogaster) were fed with an effective dose of $0.1 \%$ myricetin three hours before to the treatment with 500 $\mu \mathrm{M}$ of Rotenone (LD 50) for seven days and on $8^{\text {th }}$ day through behavioral analysis the neuroprotective effect of myricetin was investigated for motor coordination in fruit flies. Lipid peroxidation was analyzed by estimating the levels of TBARS. Oxidative stress was determined by estimating the activities of enzymatic antioxidants superoxide dismutase, catalase, and glutathione peroxidase along with the level of reduced glutathione. Dopamine level was estimated in HPLC column detected at $280 \mathrm{~nm}$ with UV detectors and degree of apoptosis was studied apoptotic marker Bcl-2, Bax, caspases- 3 and 9, cytochrome $\mathrm{c}$ and $\beta$-actin expressions in the whole body homogenate of fruit flies of experimental groups homogenized in $500 \mu \mathrm{L}$ of $0.1 \mathrm{M}$ phosphate buffers (ice cold, $\mathrm{pH}, 7.4$ ) containing 1 mmol EDTA

Results: Myricetin maintains the positive behavioral patterns against motor impairments due to the rotenone toxicity, it creates a balance in oxidant and antioxidant status, reduces the oxidative stress and inhibits apoptosis to retard neurodegeneration and maintains the dopamine level with a significant $(\mathrm{p}<0.05)$ difference compared to the rotenone treated group.

Conclusion: The flavonoid myricetin by reducing the oxidative stress, maintaining the enzymatic antioxidants status and by inhibiting apoptosis prevents the degeneration of dopaminergic neurons. The dopaminergic neurons prevention reduces the depletion of dopamine and thereby promotes the muscular coordination and psychological well being of fruit flies of experimental group. Further in depth molecular level studies are in need to explore the preventive mechanisms of myricetin in Parkinson's disease.

Keywords: Parkinson's disease, Myricetin, Rotenone, Climbing assay, Apoptosis

(C) 2017 The Authors. Published by Innovare Academic Sciences Pvt Ltd. This is an open access article under the CC BY license (http://creativecommons.org/licenses/by/4.0/) DOI: http://dx.doi.org/10.22159/ijpps.2017v9i11.19931

\section{INTRODUCTION}

Parkinson's disease (PD) is the common neurodegenerative movement disorder next to Alzheimer's disease, with multiple factors of etiology which leads to the death of dopaminergic neurons in the aged population of $60 \mathrm{y}$. The degeneration of dopaminergic neurons of substantia nigra pars compacta (SNpc) is the pathology of the disease which reduces the level of the neurotransmitter called dopamine (DA), which is involved in the locomotory event of the body. Dopamine depletion causes a negative impact on the motor circuit regulation of basal ganglia. This leads to motor impairment such as bradykinesia, muscular rigidity, tremor, and postural instability [1]. Free radical overproduction, oxidative stress, apoptosis, inflammation and abnormalities in mitochondria are critical mediators of the neuronal degeneration in PD [2]. Since the brain is sensitive to oxidative damage because of its peroxidizable fatty acid composition, consumes excess oxygen and not well enriched in antioxidant defenses. In addition, reactive oxygen species (ROS) produced enormously due to endogenous and exogenous stimuli leads to induction of signal transduction that cause mitochondrial dysfunction and cell death [3] An important hallmark of PD is Lewy's body inclusions, which are formed in dopaminergic cell aggregation area like substantia nigra (SN), dorsal nucleus of the vagus nerve and leads to the degeneration of DA neurons. Decrease in dopamine level forms the imbalance between dopaminergic and acetylcholine system which develops a series of PD symptoms [4,5]. Targeting phytochemicals and bioactive compounds from nature are significant in treating neurodegenerative diseases. Seaweeds are rich in bioactive compounds like sulfated polysaccharides, phlorotannins, flavonoids and diterpenes are having a prominent role in therapeutics research [6]. Turbinaria ornata, spiny leaf seaweed has been studied for its antioxidant, antiulcer, and wound healing, anti-inflammatory activity and hepatoprotective activities; in our previous studies, also we have elucidated the in vitro antioxidant activity it [7], free radical scavenging, antihemolysis and antiinflammatory activity of Turbinaria ornata methanolic extract [8]. In the present study neuroprotective activity of myricetin, a flavonoid isolated from Turbinaria ornata has been investigated in experimental PD models of Drosophila melanogaster.

\section{MATERIALS AND METHODS}

\section{Chemicals and reagents}

Myricetin (Isolated from Turbinaria ornata), rotenone, thiobarbituric acid (TBA), reduced glutathione and 3, 5-dithio-bisnitrobenzoic acid (DTNB), xanthine, xanthine oxidase, bovine serum albumin (BSA), primary and secondary antibodies were purchased from Sigma chemical Company, Bangalore, India. All other reagents were of analytical grade and were procured locally.

\section{Collection of samples}

The marine brown alga Turbinaria ornata was collected by hand picking from intertidal waters of the Mandapam coast (Longitude $78^{\circ}$ $8^{\prime} \mathrm{E}$, Latitude $90^{\circ} 17^{\prime} \mathrm{N}$ ) in the Gulf of Mannar during the early hours in the month of May. The algal material was identified and authenticated in BSI Coimbatore, Tamilnadu (BSI/SRC/5/23/ $2015 /$ Tech./1304). A voucher specimen was maintained in our 
research laboratory. Collected algal material was washed with sea water and then with fresh water to remove sand, salts and epiphytes.

\section{Isolation and characterization of myricetin}

The dried Turbinaria ornata seaweed materials (50g) were milled and extracted using $500 \mathrm{ml}$ of various solvents such as ethanol, methanol and water for $24 \mathrm{~h}$ by using a Soxhlet apparatus. Each filtrate was concentrated to dryness under reduced pressure using a rotary evaporator. The samples were lyophilized by using freeze dryer (Lark, Penguin Classic Plus, India) and stored in a refrigerator at $2-8^{\circ} \mathrm{C}$ for use in preliminary phytochemical analysis and subsequent experiments. The methanolic extract with more bioactive compounds used for further extraction and characterization. The flavonoids were determined by reversedphase HPLC based on the method described by Crozier et al., [9]. Flavonoid standards were morin and myricetin, an aliquot of sample extracts was loaded on the HPLC equipped with a reverse phase column. Solvents comprise deionized water (solvent A) and acetonitrile (solvent $\mathrm{B}$ ) were used. The $\mathrm{pH}$ of water was adjusted to 2.5 with trifluoroacetic acid. The flavonoid compounds were detected at $350 \mathrm{~nm}$. The column was equilibrated with $85 \%$ deionized water and 15\% acetonitrile. Then the ratio of deionized water was increased to $85 \%$ in $50 \mathrm{~min}$ followed by reducing acetonitrile to $15 \%$ in $55 \mathrm{~min}$. This ratio was maintained to $60 \mathrm{~min}$ for the subsequent analysis with flow rate at $0.6 \mathrm{ml} / \mathrm{min}$. The methanolic fraction $(40 \mathrm{~g})$ with most bioactive compounds was subjected to silica gel column chromatography $(75 \mu \mathrm{m}, 6.0 \mathrm{~cm} \times 40$ $\mathrm{cm}$ ) and eluted with methanol of increasing polarities with water (25 to $100 \%$ Methanol). The volume of each collected fraction was 50 $\mathrm{ml}$ to give 4 fractions (A, B, C, and D). Appreciable antioxidant activity was found in fraction $C$ and D. Among the two, The most active D fraction obtained (12.21 mg) was then subjected to silica gel column chromatography (Silica gel 60, 230-400 mesh, $1.0 \mathrm{~cm}$ i.d. x $20 \mathrm{~cm}$ ) and eluted with n-hexane: EtOAc, EtOAc, acetone and methanol. Compound 1 was yielded (4.2 mg) from n-hexane: EtOAc (3:7) fraction which is yellow crystals. The isolated compound was recorded on NMR-400 $\mathrm{MHz}$ and chemical shifts were recorded as $\delta$ values. The result was compared with the reference chart and possible functional groups present in the plant were determined [10].

\section{Drosophila melanogaster treatment strategies}

All the experiments were carried out in the CS strain of male Drosophila melanogaster (8-10 d old flies). They were housed in polypropylene vials with drosophila culture medium, renewed every three days. Flies were under 12/12h: light/dark cycle at around $20^{\circ} \mathrm{C}$ [11]. The culture medium was as follows; Agar 2.0g, maize powder $17.0 \mathrm{~g}$, sucrose $15.0 \mathrm{~g}$, yeast tablets $6.0 \mathrm{~g}$ and water $360 \mathrm{ml}$ were taken in a 1 litre glass beaker and heated for $1 \mathrm{~h}$ with constant stirring. After cooking, 1.0g nepagin and propionic acid (1 ml) were added and the medium $(0.1 \%$ myricetin and $500 \mu \mathrm{M}$ rotenone were added to the respective experimental groups) was then transferred to Drosophila culture vials. Then, the flies were transferred to these bottles/vials and cultured.

\section{Rotenone lethal dose study (LD 50 )}

The flies were exposed to varying concentrations $(250 \mu \mathrm{M}, 500 \mu \mathrm{M}$, $750 \mu \mathrm{M}$ and $1000 \mu \mathrm{M}$ ) of rotenone (ROT) for $7 \mathrm{~d}$ in order to assess the lethality responses to choose the effective dose for LD $_{50}$ [12].

\section{Myricetin effective dose fixation study against mortality rate}

For the preventive dose fixation of myricetin of different concentrations $0.025 \%, 0.05 \%$ and $0.1 \%$ ) was done as per the earlier findings and studies [13], against 500 $\mu \mathrm{M}$ (LD50) rotenone treatment in $7 \mathrm{~d}$ study, on the basis of reduce in mortality. $0.1 \%$ myricetin shows significant reduction in mortality compares to other two concentrations $(0.025 \%$ and $0.05 \%)$ (Data not shown) and was chosen as the active dose in further neuroprotective studies. Male flies were randomly divided into four groups with 50 flies each and were treated as given below.

\section{Experimental design}

- Group I: Normal flies with corn meal media served as control.
- Group II: These flies received ingestion of rotenone $(500 \mu \mathrm{M})$ through the media for seven consecutive days.

- Group III: These flies are pre-treated with ( $0.1 \%$ effective dose) of myricetin before three hours of rotenone treatment and then transferred to vials with rotenone $(500 \mu \mathrm{M})$ for 7 consecutive days (co-exposure).

- Group IV: These flies are treated with (0.1\% effective dose) of myricetin alone for seven days as like group III flies. (Each group contains three vials of 50 male Drosophila melanogaster)

On the end of experiment ( $8^{\text {th }}$ day), behavioral studies were performed to understand motor skill abnormalities and then flies were anesthetized. The flies were homogenized in phosphate buffer $(\mathrm{pH} 7.0)$ and centrifuged at 5000rpm for $20 \mathrm{~min}$ at $4{ }^{\circ} \mathrm{C}$ to get the supernatant which was used to estimate the levels of TBARS and GSH, and activities of SOD, CAT, and GPx.

\section{Behavioural assessment studies}

To assess the motor performance, muscular coordination, and signs of Parkinsonism the following behavioral assessment methods were employed as per the earlier studies [14], with slight modifications. Behavioural changes in PD depend on the degree of neuronal dysfunction. Behavioral analysis in models of PD is significant in investigating the relationship among neuronal degeneration and the effectiveness of the therapy and brain recovery process [15].

\section{Open field test}

A Single fly was put into a Petri dish (Open field chamber) marked with the grid of $1 \mathrm{~cm}$ square. The fly is permitted in for $10 \mathrm{~min}$. After which the locomotor activities will be absorbed for first two minutes and last two minutes of a 10 min period. Locomotion absorbed in first two minutes is exploratory and the last two minutes is considered as basal locomotion (the remaining 6-8 $\mathrm{min}$ will be considered as the sufficient time for flies to acclimatize to the environment). The number of grid lines crossed represents the measure of locomotor activity [16]. The flies of each group were randomly allowed for the open field test.

\section{Climbing assay}

Climbing assay was done as per the protocols of previous study [17]. Briefly, in climbing assay, experimental flies were placed in an empty glass tube $(25 \mathrm{~cm}$ height and $2 \mathrm{~cm}$ diameter). After a 10 min rest period, the flies were tapped to the bottom of the glass tube, and the number of flies able to climb in $60 \mathrm{~s}$ was recorded at each interval of time. The assay was replicated three times at $5 \mathrm{~min}$ intervals. The scores are the mean of the numbers of flies at the top and at the bottom, expressed as percentages of the total number of flies.

\section{Analysis of dopamine concentrations by HPLC}

The flies from each group were homogenized in $500 \mu \mathrm{L}$ of $0.1 \mathrm{M}$ phosphate buffers (ice cold, $\mathrm{pH}, 7.4$ ) containing 1 mmol EDTA. Followed by centrifugation at 2,500×g for $10 \mathrm{~min}$, the supernatant of the homogenization was filtered and injected into the HPLC column detected at $280 \mathrm{~nm}$ with UV detectors. The flow rate of a mobile phase consisting of $0.2 \%$ aqueous trifluoroacetic acid and methanol $(70: 30 \mathrm{v} / \mathrm{v})$ was maintained flow rate of $1 \mathrm{ml} / \mathrm{min}$ [18].

\section{Lipid peroxidation products and enzymatic antioxidants activity}

- Estimation of TBARS: The method of Utley et al., (1967) was used for the estimation of lipid peroxidation [19].

- Estimation of total protein: Protein concentration in the whole body fly homogenate was determined after trichloroacetic acid precipitation as described previously [20], using bovine serum albumin as the standard.

- Estimation of Superoxide dismutase: (SOD) activity was measured by the method of Beauchamp and Fridovich (1971) [21].

- Estimation of Catalase: Catalase activity was assayed by the method of Sinha (1972) [22].

- Estimation of reduced glutathione: Reduced glutathione (GSH) was determined by the method of Jollow et al., (1974) [23]. 
- Estimation of glutathione peroxidase: Determination of Glutathione peroxidase (GPx) activity measured according to the procedure described by Mohandas et al., (1984) [24].

\section{Western blot analysis}

A total volume of $30 \mu \mathrm{g}$ of isolated protein from drosophila homogenate was loaded per lane. The separated proteins were blotted onto a PVDF membrane by semi-dry transfer (BIO-RAD). After blocking with $5 \%$ non fat milk in TBS, the membranes were then incubated with various antibodies: Bcl-2, Bax, caspases-3 and 9, cytochrome $\mathrm{c}$ and $\beta$-actin. The following dilutions were used for Bcl2 and Bax (1:500), cytochrome c, caspases-3 and 9 (1:1000), and $\beta$ actin (1:2000). After primary antibody incubation, the membranes were incubated with secondary antibody at a concentration of 1:2000. Then the membranes were washed with Tris-buffered saline and $0.05 \%$ Tween-20 thrice for $10 \mathrm{~min}$ interval, after extensive washes in TBST, the bands were visualized by treating the membranes with 3, 3'-diaminobenzidine tetra hydrochloride (Western blot detection reagent, Sigma, USA). Densitometry was done using 'Image J' analysis software [25].

\section{Statistical analysis}

All numerical results were expressed as mean \pm standard deviation (SD) and analyzed using the statistical package for the social sciences (SPSS) IBM SPSS Statistic. One-way analysis of variance (ANOVA) was conducted for statistical comparison of study whereby $\mathrm{p}<0.05$ being the criterion for statistical significance. The significant treatment means were further subjected to Duncan's post hoc test.

\section{RESULTS}

Fig. 1, depicts the Rotenone concentration of $\mathrm{LD}_{50}$. Among the different doses $500 \mu \mathrm{M}$ of Rotenone induced the $50 \%$ of mortality and so it was fixed as the effective dose of rotenone concentration to induce the Parkinsonism in Drosphila melanogaster.

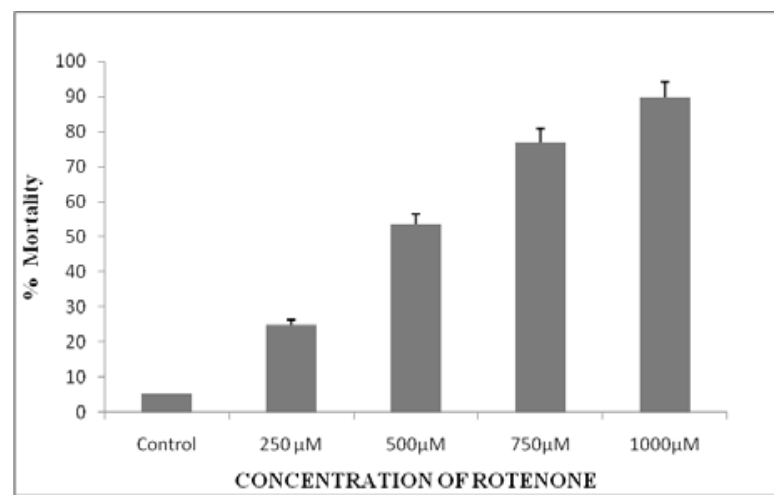

Fig. 1: Shows the rotenone exposure study for $\mathrm{LD}_{50}$ of rotenone concentration through percentage of mortality induction in experimental flies

Fig. 2a shows the exploration and locomotors activity of experimental groups in open field test through number of squares the flies crossed. The group II rotenone treated drosophila crossed only 6 squares, exhibits frequent immobility and shows more time consumption to acclimatize, reduced exploration and basal locomotion in the open field chamber, compared to control group I and myricetin alone treated group IV (crossed 25 and 26 squares respectively). Group III flies (myricetin and rotenone treated) crossing about 18 squares and exhibit occasional immobility as the sign of appreciable exploration, acclimatization and basal locomotion compared with group II rotenone treated flies.

Fig. $2 \mathrm{~b}$ shows the climbing assay of experimental groups in $25 \mathrm{~cm}$ long glass tubes. The group II rotenone treated flies consumed more time to climb the $25 \mathrm{~cm}$ glass tube and only about $38 \%$ flies managed to reached the top compared to group I control and group IV myricetin alone treated groups in which about $92 \%$ and $91 \%$ of flies have reached top respectively. Group III flies (myricetin and rotenone treated) shown appreciable reduction in time consumption and about $77 \%$ of flies climbed to top compared to group II rotenone treated flies.
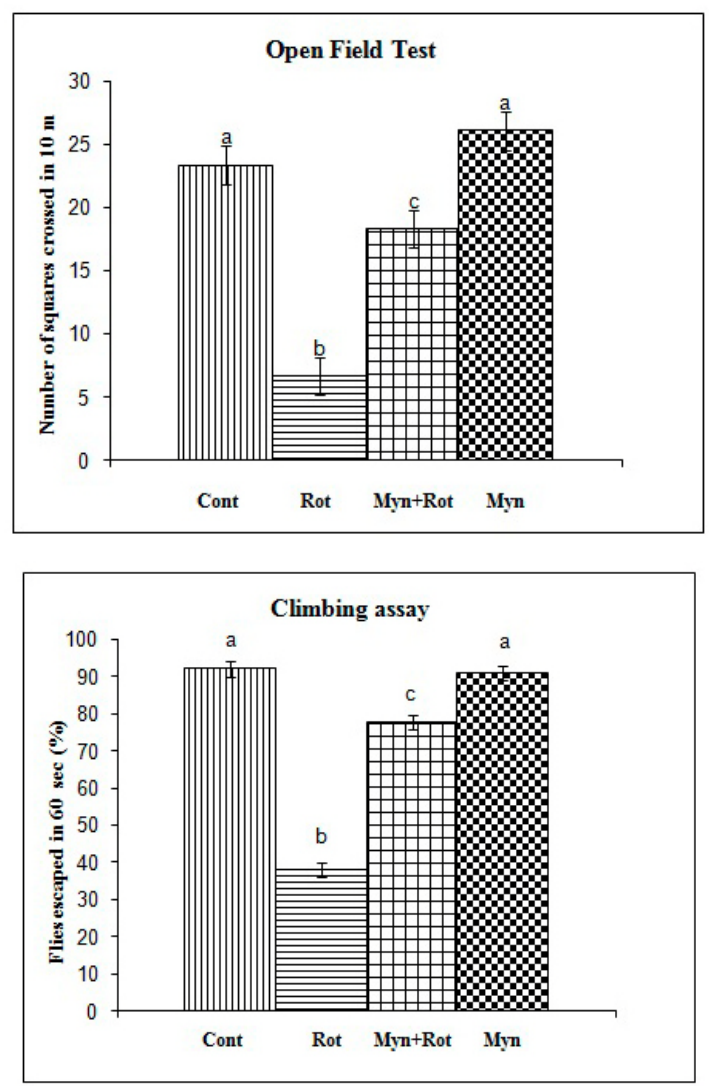

Fig. 2: ( $a$ and $b$ ). Shows the time taken in Open field test (Number of squares crossed) for exploring, acclimatization and basal locomotors activity in control and experimental groups and Climbing assay shows the time taken to climb $25 \mathrm{~cm}$ long glass tube in control and experimental groups in 60 s (Values are given as mean \pm SD for 3 vials of drosophila 50 flies each group. Values not sharing a common superscript letter differs significantly at $\mathbf{p}<0.05$ (DMRT))

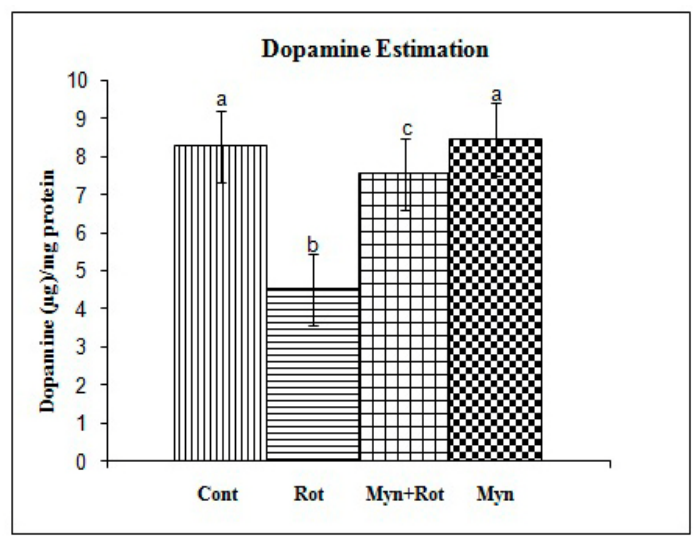

Fig. 3: Dopamine levels in the whole body homogenate of control and experimental groups. (Values are given as mean \pm SD for 3 vials of drosophila 50 flies each group. Values not sharing a common superscript letter differ significantly at p<0.05 (DMRT)) 
Fig. 3 elucidates the dopamine level in control and experimental groups. The group II rotenone treated Drosophila homogenate samples shows decreased concentration of about $4.50 \mu \mathrm{g}$ of dopamine compared to the control group I and myricetin alone treated group IV (about $8.27 \mu \mathrm{g}$ and $8.45 \mu \mathrm{g}$ respectively). In group III, myricetin maintains the dopamine concentration $(7.53 \mu \mathrm{g})$ much similar to control and group IV against the impact of rotenone Clearly indicates the prevention of rotenone induced neurodegeneration of DA (dopaminergic neurons) neurons.

Fig. 4 and table 1 shows the levels of TBARS and enzymatic antioxidant activities. The group II rotenone treated Drosophila homogenate samples shows increased TBARS level, SOD, CAT, and GPx activity; and decreased GSH level compared to the control group I and myricetin alone treated group IV. In group III, myricetin prevents the impact of rotenone in inducing free radicals and oxidative stress and maintains the TBARS level, activities of SOD, CAT, GPx and GSH level near normal.

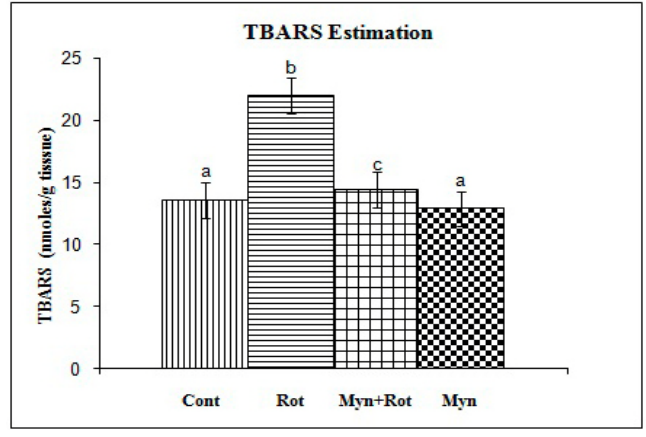

Fig. 4: Changes in the levels of TBARS in whole body homogenate of control and experimental groups. Values are given as mean \pm SD for 3 vials of drosophila 50 flies each group. Values not sharing a common superscript letter differ significantly at $\mathbf{p}<0.05$ (DMRT)

Table 1: Changes in the activities of SOD, CAT, GPx and level of GSH in control and experimental groups.

\begin{tabular}{|c|c|c|c|c|}
\hline Groups & 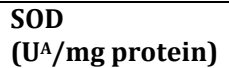 & $\begin{array}{l}\text { CAT } \\
\text { (U⿱一土㇒/mg protein) }\end{array}$ & $\begin{array}{l}\text { GPX } \\
\text { UC/mg protein) }^{C} / \mathbf{m} \text {. }\end{array}$ & $\begin{array}{l}\text { GSH } \\
\text { (mg/g tissue) }\end{array}$ \\
\hline Control & $7.16 \pm 0.82^{\mathrm{a}}$ & $3.22 \pm 0.31^{\mathrm{a}}$ & $7.15 \pm 0.60^{\mathrm{a}}$ & $2.14 \pm 0.32^{\mathrm{a}}$ \\
\hline Rotenone & $11.31 \pm 0.21^{\mathrm{b}}$ & $6.42 \pm 0.32^{b}$ & $12.34 \pm 0.32^{\mathrm{b}}$ & $1.09 \pm 0.11^{b}$ \\
\hline $\begin{array}{l}\text { Myricetin+ } \\
\text { Rotenone }\end{array}$ & $8.69 \pm 0.35^{c}$ & $4.37 \pm 0.23^{c}$ & $8.49 \pm 0.43^{c}$ & $2.01 \pm 0.15^{c}$ \\
\hline Myricetin & $7.68 \pm 0.16^{a}$ & $3.02 \pm 0.12^{\mathrm{a}}$ & $7.27 \pm 0.12^{\mathrm{a}}$ & $2.27 \pm 0.06^{\mathrm{a}}$ \\
\hline
\end{tabular}

(Values are given as mean \pm SD for 3 vials of drosophila 50 flies each group. Values not sharing a common superscript letter differ significantly at $\mathrm{p}<0.05$ (DMRT). $\mathrm{A}=$ enzyme required for $50 \%$ inhibition of NBT reduction. $\mathrm{B}=\mu$ moles of $\mathrm{H}_{2} \mathrm{O}_{2}$ utilized/min. $\mathrm{C}=\mu \mathrm{moles}$ of glutathione utilized/min).

Rotenone treatment $(500 \mu \mathrm{M})$ leads to decreased expression levels of anti-apoptotic B-cell CLL/lymphoma 2 (Bcl-2), but it increased the expression levels of pro-apoptotic Bcl-2-associated X protein (Bax). myricetin pretreatment $(0.1 \%)$ retains the normal expression of $\mathrm{Bcl}-2$ and Bax (fig. 5a). Treatment with rotenone $(500 \mu \mathrm{M})$ increased expression levels of cyt c, caspases 3 and 9. myricetin pretreatment significantly suppressed the enhanced cyt c, caspases 3 and 9 expressions and prevents the apoptosis due to rotenone toxicity (fig. 5b).

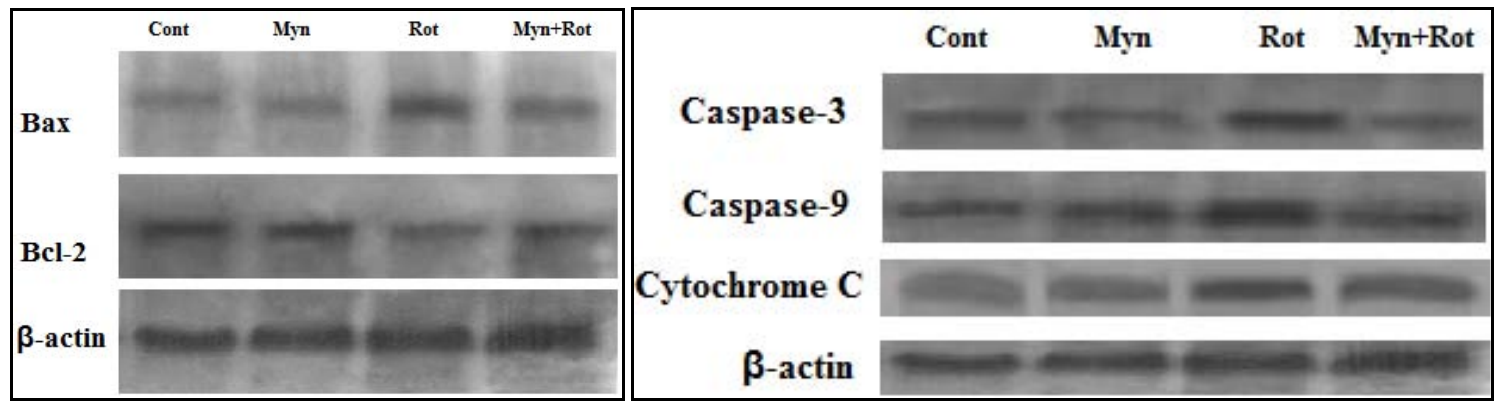

Fig. 5: ( $a$ and b) shows the antiapoptotic effect of myricetin on rotenone induced Bax, Bcl-2, cyt c, caspases 3 and 9

\section{DISCUSSION}

Parkinson's disease occurs due to oxidative stress, mitochondrial dysfunction and DA neurons degeneration. Rotenone, an environmental toxin and a complex I inhibitor of ETC induce mortality, oxidative stress and mitochondrial dysfunction in DA neurons of Drosophila melanogaster. In the rotenone exposure study for $\mathrm{LD}_{50}$ at a concentration of $500 \mu \mathrm{M}$ there is $53 \%$ of mortality of flies occurred and the mortality rate is concentration dependent. In our study rotenone at a concentration of $500 \mu \mathrm{M}$ renders deficits in locomotor activity and muscular coordination which is well understood by our findings in the open field test and climbing assay.

Movement disorders and lack of muscular coordination is the important pathologic features in $\mathrm{PD}$, due to degeneration of pigmented DA neurons in SN (60-70\% of the neuronal loss in SN and striatal DA content is reduced by $80 \%$ ), that triggers the symptoms such as akinesia, rigidity, resting tremor, gait and postural abnormalities [26]. Group II flies subjected to $500 \mu \mathrm{M}$ of Rotenone in our study leads to deficits in exploration activity and basal locomotor activity. They also took more time for acclimatization and show frequent immobility. This is well elucidated by the increase in the time consumption and decrease in exploration of squares, whereas controls group I and group III (myricetin and rotenone treated) and group IV (myricetin) shows appreciable locomotion and explores more squares with occasional immobility in open field test. Similarly deficits in muscular coordination are shown by the percentage of flies climbing to the top in $60 \mathrm{~s}$ in the $25 \mathrm{~cm}$ glass tube. In group II ROT treated only $38 \%$ of flies climb to the top compared to control (92\%), In Group III (myricetin and rotenone treated) nearly $77 \%$ of flies climbed up to the top, which clearly shows the preventive role of myricetin against rotenone in neurodegeneration and promotes proper muscular coordination.

The important neuro-transmitter dopamine which is synthesized in dopaminergic neurons (DA) plays a vital role in muscular 
coordination. Degeneration of pigmented DA neurons in the Substantia nigra (SN) and loss of striatal dopamine is the hallmark of PD. In the present study group II rotenone treated shows $4.50 \mu \mathrm{g}$ of dopamine compared to the control group I $(8.27 \mu \mathrm{g}$ of dopamine) which clearly indicates the degeneration of DA neurons by rotenone. Myricetin in group III significantly prevents the DA neurons and thereby shows increased dopamine concentration of $7.53 \mu \mathrm{g}$ compared to the Group II flies. myricetin alone treated group IV shows nearly similar concentration of dopamine $(8.45 \mu \mathrm{g})$ like group I control. The results of behavioral study and levels of dopamine are very much supported by earlier different study [27].

The antioxidative property of myricetin is well elucidated by the levels of TBARS and GSH and activities of enzymatic antioxidants in control and experimental groups. The level of increased TBARS, decreased GSH levels and increased activities of SOD and catalase were observed in Rotenone treated group II as compared to control group I (fig. 1 and table 2). However, a decrease in TBARS, increase in GSH level and significant reduction in the activities of SOD and catalase were observed in group III (myricetin pretreated and rotenone treated), is the proof of antioxidant property of myricetin against Rotenone neurotoxicity. The levels of TBARS, GSH and activities of enzymatic antioxidants in group IV were normal and similar to group I control. Our results are well supported by previous findings in different experimental PD [28].

\section{CONCLUSION}

In the present study, a flavonoid myricetin isolated from Turbinaria ornata with the richest source of bioactive compounds was investigated for its neuroprotective activity in Drosophila melanogaster model. Our study results show that myricetin promotes the antioxidant and oxidant balance thereby reduced the oxidative stress. By reducing oxidative stress, prevents the DA neurodegeneration and maintains the dopamine level. Myricetin by inhibiting DA neurons death promotes the muscular coordination and psychological well being of Drosophila melanogaster. Further molecular studies in cell line and animal models are in need to explore the preventive mechanisms of myricetin in Parkinson's disease.

\section{ACKNOWLEDGEMENT}

The authors are thankful to Dr. T. Manivasagam, Department of Biochemistry and Biotechnology of Annamalai University; and Department of Biochemistry of Rev. Jacob Memorial Christian College for necessary assistance.

\section{AUTHORS CONTRIBUTION}

Conceived and designed the experiments: Vijayraja Dhanraj, Tamilarasan Manivasagam and Jeyaprakash karuppaiah. Performed the experiments: Vijayraja Dhanraj. Analyzed the data: Tamilarasan Manivasagam and Jeyaprakash karuppaiah. Contributed reagents/ materials/analysis tools: Tamilarasan Manivasagam and Jeyaprakash karuppaiah. Manuscript preparation and correction and Correspondence: Vijayraja Dhanraj and Jeyaprakash karuppaiah respectively.

\section{CONFLICT OF INTERESTS}

Declared none

\section{REFERENCES}

1. Khadira Sereen A, Vijayalakshmi K, Priya Nagappan, Shinu Balima. Effect of sesamol in association with folic acid on 6OHDA induced Parkinsonian animals-biochemical, neurochemical and histopathological evidence. Asian J Pharm Clin Res 2017;10:46-50.

2. Vera Dias, Eunsung J, Maral Mouradian M. The role of oxidative stress in parkinson's disease. J Parkinson's Dis 2013;3:461-91.

3. Suryakanta Pany, Abhisek Pal, Pratap Kumar Sahu. Neuroprotective effect of quercetin in neurotoxicity induced rats: role of neuroinflammation in neurodegeneration. Asian J Pharm Clin Res 2014;7:152-6.

4. Uchihara T, Giasson BI. Propagation of alpha-synuclein pathology: hypotheses discoveries, and yet unresolved questions from experimental and human brain studies. Acta Neuropathol 2016;131:49-73.

5. Butterfield DA, Perluigi M, Reed T, Muharib T, Hughes $\mathrm{CP}$, Robinson RA, et al. redox proteomics in selected neurodegenerative disorders: from its infancy to future applications. Antioxid Redox Signal 2012;17:1610-55.

6. Mariya V, Vinoth S. Biomedical and pharmacological significance of marine macro algae-review. Indian J Mar Sci 2013;42:527-37.

7. Vijayraja D, Jeyaprakash K. Phytochemical analysis, in vitro antioxidant and anti-hemolysis activity of Turbinaria ornata (turner) J. Agardh Int J Adv Res Sci Eng Technol 2015;12:45-9.

8. Vijayraja D, Jeyaprakash K. Phytochemical analysis, in vitro DPPH free radical scavenging, anti-hemolysis and antiinflammatory activities of Turbinaria ornata (Turner) J. Agardh World J Pharm Pharm 2016;5:1231-8.

9. Crozier A, Lean MEJ, McDonald MS, Black C. Quantitative analysis of the flavonoid content of commercial tomatoes, onions, lettuce and celery. J Agric Food Chem 1997;45:590-5.

10. Das S, Ganapaty S. Phytochemical evaluation of roots of polygonum viscosum buch-ham. Indian J Pharm Sci 2015;77:352-6.

11. Bilen J, Bonini NM. Drosophila as a model for human neurodegenerative disease. Annu Rev Genet 2005;39:153-71.

12. Hosamani R, Ramesh SR, Muralidhara. Attenuation of rotenoneinduced mitochondrial oxidative damage and neurotoxicty in Drosophila melanogaster supplemented with creatine. Neurochem Res 2010;35:1402-12.

13. Jeibmann A, Paulus W. Drosophila melanogaster as a model organism of brain diseases. Int J Mol Sci 2009;10:407-40.

14. Jimenez-Del-Rio $M$, Daza-Restrepo A, Velez-Pardo C. The cannabinoid CP55, 940 prolongs survival and improves locomotor activity in Drosophila melanogaster against paraquat: implications in Parkinson's disease. Neurosci Res 2008;61:404-11.

15. Anandhan A, Tamilselvam K, Vijayraja D. Resveratrol attenuates oxidative stress and improves behaviour in 1-methyl-4-phenyl-1,2,3,6-tetrahydropyridine (MPTP) challenged mice. Ann Neurosci 2010;17:113-9.

16. Hirth F. Drosophila melanogaster in the study of human neurodegeneration. CNS Neurol Disord Drug Targets 2010;9:504-23.

17. Liu Z, Wang X, Yu Y, Li X, Wang T, Jiang H, et al. A drosophila model for LRRK2-linked parkinsonism. Proc Natl Acad Sci USA 2008;105:2693-8.

18. Dalpiaz A, Filosa R, de Caprariis P, Conte G, Bortolotti F, Biondi C, et al. Molecular mechanism involved in the transport of a prodrug dopamine glycosyl conjugate. Int J Pharm 2007;336:133-9.

19. Utley HC, Bernheim F, Hochslein P. Effect of sulfhydryl reagent on peroxidation in microsome. Arch Biochem Biophys 1967;118:29-32.

20. Lowry $\mathrm{OH}$, Rosebrough NJ, Farr AL, Randall RJ. Protein measurement with the Folin phenol reagent. J Biol Chem 1951;193:265-75.

21. Beauchamp C, Fridovich I. Superoxide dismutase: improved assays and an assay applicable to acrylamide gels. Anal Biochem 1971;44:276-87.

22. Sinha AK. Colorimetric assay of catalase. Anal Biochem 1972;47:389-94.

23. Jollow DJ, Mitchell JR, Zampagloine N, Gillete JR. Bromobenzene-induced liver necrosis: protective role of glutathione and evidence for 3, 4 bromobenzeneoxide as the hepatotoxic intermediate. Pharmacology 1974;11:151-69.

24. Mohandas J, Marshall JJ, Duggin GG, Horvath JS, Tiller DJ. Differential distribution of glutathione and glutathione related enzymes in rabbit kidneys: Possible implication in analgesic neuropathy. Cancer Res 1984;44:5086-91.

25. Tamilselvam K, Braidy N, Manivasagam T, Essa MM, Prasad NR, Karthikeyan S, et al. Neuroprotective effects of hesperidin, a plant flavanone, on rotenone-induced oxidative stress and apoptosis in a cellular model for Parkinson's disease. Oxid Med Cell Longev 2013:102741. Doi:10.1155/2013/102741.

26. Joao Massano, Kailash $\mathrm{P}$ Bhatia. Clinical approach to Parkinson's disease: features, diagnosis, and principles of management. Cold Spring Harbor Perspect Med 2012;2:1-15. 
27. Manjunath MJ, Muralidhara. Standardized extract of Withania somnifera (Ashwagandha) markedly offsets rotenoneinduced locomotor deficits, oxidative impairments and neurotoxicity in Drosophila melanogaster. J Food Sci Technol 2015;52:1971-81.
28. Nataraj J, Manivasagam T, Thenmozhi AJ, Essa MM. Lutein protects dopaminergic neurons against MPTP-induced apoptotic death and motor dysfunction by ameliorating mitochondrial disruption and oxidative stress. Nutr Neurosci 2016;19:237-46. 